\title{
Pseudo-potential treatment of two aligned dipoles under external harmonic confinement
}

\author{
K. Kanjilal, ${ }^{1}$ John L. Bohn, ${ }^{2}$ and D. Blume ${ }^{1,3}$ \\ ${ }^{1}$ Department of Physics and Astronomy, Washington State University, Pullman, WA 99164-2814 \\ ${ }^{2}$ JILA, NIST and Department of Physics, University of Colorado, Boulder, CO 80309-0440 \\ ${ }^{3}$ INFM-BEC, Dipartimento di Fisica, Università di Trento, Sommarive 4, I-38050 Povo, Italy
}

\begin{abstract}
Dipolar Bose and Fermi gases, which are currently being studied extensively experimentally and theoretically, interact through anisotropic, long-range potentials. Here, we replace the long-range potential by a zero-range pseudo-potential that simplifies the theoretical treatment of two dipolar particles in a harmonic trap. Our zerorange pseudo-potential description reproduces the energy spectrum of two dipoles interacting through a shapedependent potential under external confinement very well, provided that sufficiently many partial waves are included, and readily leads to a classification scheme of the energy spectrum in terms of approximate angular momentum quantum numbers. The results may be directly relevant to the physics of dipolar gases loaded into optical lattices.
\end{abstract}

PACS numbers: $34.50 .-\mathrm{s}, 34.10 .+\mathrm{x}$

\section{INTRODUCTION}

Many-body systems with dipolar interactions have attracted a lot of attention recently. Unlike the properties of ultracold atomic alkali vapors, which can be described to a very good approximation by a single scattering quantity (the $s$ wave scattering length), those of dipolar gases additionally depend on the dipole moment. This dipole moment can be magnetic, as in the case of atomic $\mathrm{Cr}$ [1, 2], or electric, as in the case of heteronuclear molecules such as $\mathrm{OH}[3,4]$, $\mathrm{KRb}$ [5] or RbCs [6]. Furthermore, dipolar interactions are long-ranged and anisotropic, giving rise to a host of novel many-body effects in confined dipolar gases such as rotonlike features [7, 8, 9] and rich stability diagrams $[10,11,12$, 13, 14, 15, 16, 17]. The physics of dipolar gases loaded into optical lattices promises to be particularly rich. For example, this setup constitutes the starting point for a range of quantum computing schemes $[18,19,20,21]$. Additionally, a variety of novel quantum phases have already been predicted to arise [22, 23, 24, 25]. Currently, a number of experimental groups are working towards loading dipolar gases into optical lattices.

This paper investigates the physics of doubly-occupied optical lattice sites in the regime where the tunneling between neighboring sites and the interactions with dipoles located in other lattice sites can be neglected. In this case, the problem reduces to treating the interactions between two dipoles in a single lattice site. Assuming that the lattice potential can be approximated by a harmonic potential, the center of mass motion separates and the problem reduces to solving the Schrödinger equation for the relative distance vector $\vec{r}$ between the two dipoles. The interaction between the two aligned dipoles is angle-dependent and falls off as $1 / r^{3}$ at large interparticle distances. In this work, we replace the shape-dependent interaction potential by an angle-dependent zero-range pseudo-potential, which is designed to reproduce the scattering properties of the full shape-dependent interaction potential, and derive an implicit eigenequation for two interacting identical bosonic dipoles and two interacting identical fermionic dipoles analytically.

Replacing the full interaction potential or a shape- dependent pseudo-potential by a zero-range pseudopotential [26, 27, 28, 29, 30, 31, 32] often allows for an analytical description of ultracold two-body systems in terms of a few key physical quantities. Here we show that the eigenequation for appropriately chosen zero-range pseudo-potentials reproduces the energy spectrum of two dipoles under harmonic confinement interacting through a shape-dependent model potential; that the applied zerorange treatment readily leads to an approximate classification scheme of the energy spectrum in terms of angular momentum quantum numbers; and that the proposed pseudo-potential treatment breaks down when the characteristic length of the dipolar interaction becomes comparable to the characteristic length of the external confinement. The detailed understanding of two interacting dipoles obtained in this paper will guide optical lattice experiments and the search for novel many-body effects.

Section Пintroduces the Hamiltonian under study and discusses the anisotropic zero-range pseudo-potential that is used to describe the scattering between two interacting dipoles. In Sec. IIII we derive an implicit eigen equation for two dipoles under external spherical harmonic confinement interacting through the zero-range pseudo-potential and show that the resulting eigenenergies agree well with those obtained for a shape-dependent model potential. Finally, Sec.[V]concludes.

\section{SYSTEM UNDER STUDY AND ANISOTROPIC PSEUDO-POTENTIAL}

Within the mean-field Gross-Pitaevskii formalism, the interaction between two identical bosonic dipoles, aligned along the space-fixed $\hat{z}$-axis by an external field, has been successfully modeled by the pseudo-potential $V_{p p}(\vec{r})[11]$,

$$
V_{p p}(\vec{r})=\frac{2 \pi \hbar^{2}}{\mu} a_{00} \delta(\vec{r})+d^{2} \frac{1-3 \cos ^{2} \theta}{r^{3}} .
$$

Here, $\mu$ denotes the reduced mass of the two-dipole system, $d$ the dipole moment, and $\theta$ the angle between $\hat{z}$ and the relative distance vector $\vec{r}$. The $s$-wave scattering length $a_{00}$ de- 
pends on both the short- and long-range parts of the true interaction potential. The second term on the right hand side of Eq. (1) couples angular momentum states with $l=l^{\prime}(l>0)$ and $\left|l-l^{\prime}\right|=2$ (any $l, l^{\prime}$ ). For identical fermions, $s$-wave scattering is absent and the interaction is described, assuming the long-range dipole-dipole interaction is dominant, by the second term on the right hand side of Eq. (1).

Our goal in this paper is to determine the eigenequation of two identical bosonic dipoles and two identical fermionic dipoles under external spherically harmonic confinement with angular trapping frequency $\omega$ analytically. The Schrödinger equation for the relative position vector $\vec{r}$ reads

$$
\left[H_{0}+V_{\text {int }}(\vec{r})\right] \psi(\vec{r})=E \psi(\vec{r}),
$$

where the Hamiltonian $H_{0}$ of the non-interacting harmonic oscillator is given by

$$
H_{0}=-\frac{\hbar^{2}}{2 \mu} \nabla_{\vec{r}}^{2}+\frac{1}{2} \mu \omega^{2} r^{2} .
$$

In Eq. (2), $V_{\text {int }}(\vec{r})$ denotes the interaction potential. The pseudo-potential $V_{p p}(\vec{r})$ cannot be used directly in Eq. (2) since both parts of the pseudo-potential lead to divergencies. The divergence of the $\delta$-function potential arises from the singular $1 / r$ behavior at small $r$ of the spherical Neumann function $n_{0}(r)$, and can be cured by introducing the regularization operator $\frac{\partial}{\partial r} r$ [27]. Curing the divergence of the long-ranged $1 / r^{3}$ term of $V_{p p}$ is more involved, since it couples an infinite number of angular momentum states, each of which gives rise to a singularity in the $r \rightarrow 0$ limit. The nature of each of these singularities depends on the quantum numbers $l$ and $l^{\prime}$ coupled by the pseudo-potential, and hence has to be cured separately for each $l$ and $l^{\prime}$ combination.

In this work, we follow Derevianko [33, 34] and cure the divergencies by replacing $V_{p p}(\vec{r})$ with a regularized zero-range potential $V_{p p, r e g}(\vec{r})$, which contains infinitely many terms,

$$
V_{p p, r e g}(\vec{r})=\sum_{l l^{\prime}} V_{l l^{\prime}}(\vec{r}) \text {. }
$$

The sum in Eq. (4) runs over $l$ and $l^{\prime}$ even for identical bosons, and over $l$ and $l^{\prime}$ odd for identical fermions. For $l \neq l^{\prime}, V_{l l^{\prime}}$ and $V_{l^{\prime} l}$ are different and both terms have to be included in the sum. In Sec. IIII we apply the pseudo-potential to systems under spherically symmetric external confinement. For these systems, the projection quantum number $m$ is a good quantum number, i.e., the energy spectrum for two interacting dipoles under spherically symmetric confinement can be solved separately for each allowed $m$ value. Consequently, a separate pseudo-potential can be constructed for each $m$ value. In the following, we restrict ourselves to systems with vanishing projection quantum number $m$; the generalization of the pseudo-potential to general $m$ is discussed at the end of this section. The $V_{l l^{\prime}}$ are defined through their action on an arbitrary $\vec{r}$-dependent function $\Phi(\vec{r})[33,34]$,

$$
\begin{gathered}
V_{l l^{\prime}}(\vec{r}) \Phi(\vec{r})=g_{l l^{\prime}} \frac{\delta(r)}{r^{l^{\prime}+2}} Y_{l^{\prime} 0}(\theta, \phi) \times \\
{\left[\frac{\partial^{2 l+1}}{\partial r^{2 l+1}} r^{l+1} \int Y_{l 0}(\theta, \phi) \Phi(\vec{r}) d \Omega\right]_{r \rightarrow 0}}
\end{gathered}
$$

with

$$
g_{l l^{\prime}}=\frac{\hbar^{2}}{2 \mu} \frac{a_{l l^{\prime}}}{k^{l+l^{\prime}}} \frac{(2 l+1) ! !\left(2 l^{\prime}+1\right) ! !}{(2 l+1) !},
$$

where $k$ denotes the relative wave vector, $k=\sqrt{2 \mu E / \hbar^{2}}$, and the $a_{l l^{\prime}}$ generalized scattering lengths. Since we are restricting ourselves to $m=0$, the $V_{l l^{\prime}}$ are written in terms of the spherical harmonics $Y_{l m}$ with $m=0$. When applying the above pseudopotential we treat a large number of terms in Eq. (4), and do not terminate the sum after the first three terms as done in Refs. [33, 34, 35]. We note that the non-Hermiticity of $V_{p p, r e g}$ does not lead to problems when determining the energy spectrum; however, great care has to be taken when calculating, e.g., structural expectation values [36].

To understand the functional form of the zero-range pseudo-potential defined in Eqs. (4) through (6), let us first consider the piece of Eq. (5) in square brackets. If we decompose the incoming wave $\Phi(\vec{r})$ into partial waves,

$$
\Phi(\vec{r})=\sum_{n_{i} l_{i} m_{i}} c_{n_{i} l_{i} m_{i}} Q_{n_{i} l_{i}}(r) Y_{l_{i} m_{i}}(\theta, \phi),
$$

where the $c_{n_{i} l_{i} m_{i}}$ denote expansion coefficients and the $Q_{n_{i} l_{i}}$ radial basis functions, the spherical harmonic $Y_{l 0}$ in the integrand of $V_{l l^{\prime}}$ acts as a projector or filter. After the integration over the angles, only those components of $\Phi(\vec{r})$ that have $l_{i}=l$ and $m_{i}=0$ survive. The operator $\frac{\partial^{2 l+1}}{\partial r^{2 l+1}} r^{l+1}$ in Eq. (5) is designed to then first cure the $r^{-l-1}$ divergencies of the $Q_{n_{i}}$, which arise in the $r \rightarrow 0$ limit, and to then second "extract" the coefficients of the regular part of the $Q_{n_{i} l}(r)$ that go as $r^{l}$ [27]. Alltogether, this shows that the square bracket in Eq. (5) reduces to a constant when the $r \rightarrow 0$ limit is taken. To understand the remaining pieces of the pseudo-potential, we multiply Eq. (5) from the left with $Q_{n_{o} l_{o}}^{*} Y_{l_{o} m_{o}}^{*}$ and integrate over all space. The spherical harmonic $Y_{l^{\prime} 0}$ in Eq. (5) then ensures that the integral is only non-zero when $l^{\prime}=l_{o}$ and $m_{o}=0$. When performing the radial integration, the $\delta(r) / r^{l^{\prime}}$ term ensures that the coefficients of the regular part of the $Q_{n_{o} l_{o}}$ that go as $r^{l_{o}}$ are being extracted (note that the remaining $1 / r^{2}$ term cancels the $r^{2}$ in the volume element).

Alltogether, the analysis outlined in the previous paragraph shows that the functional form of $V_{l l^{\prime}}$ ensures that the divergencies of the radial parts of the incoming and outgoing wave is cured in the $r \rightarrow 0$ limit and that the $l$ th component of the incoming wave is scattered into the $l^{\prime}$ th partial wave. The sum over all $l$ and $l^{\prime}$ values in Eq. (4) guarantees that any state with quantum number $l$ can be coupled to any state with quantum number $l^{\prime}$, provided the corresponding generalized scattering length $a_{l l^{\prime}}$ is non-zero. We note that the regularized pseudo-potential given by Eqs. (4) through (6) is only appropriate if the external confining potential in Eq. (3) has spherical symmetry [37]. Generalizations of the above zero-range pseudo-potential, aimed at treating interacting dipoles under elongated confinement, require the regularization scheme to be modified to additionally cure divergencies of cylindrically symmetric wave functions. These extensions will be subject of future studies. 
We now discuss the generalized scattering lengths $a_{l l^{\prime}}$, which determine the scattering strengths of the $V_{l l^{\prime}}$. The $a_{l l^{\prime}}$ have units of length and are defined through the K-matrix elements $K_{l m}^{l^{\prime} m^{\prime}}$ [38],

$$
a_{l l^{\prime}}=\lim _{k \rightarrow 0} \frac{-K_{l 0}^{l^{\prime} 0}(k)}{k}
$$

for $m=0$. The scattering lengths $a_{l l^{\prime}}$ and $a_{l^{\prime} l}$ are identical because the K-matrix is symmetric. In general, the scattering lengths $a_{l l^{\prime}}$ have to be determined from the K-matrix elements for the "true" interaction potential, which contains the longrange dipolar and a short-ranged repulsive part, of two interacting dipoles. As discussed further in Sec. III an approach along these lines is used to obtain the squares shown in Fig. 3.

Alternatively, it has been shown that the K-matrix elements (except for $K_{00}^{00}$, see below) for realistic potentials, such as for the $\mathrm{Rb}-\mathrm{Rb}$ potential in a strong electric field [11] or an $\mathrm{OH}$ $\mathrm{OH}$ model potential [16], are approximated with high accuracy by the K-matrix elements for the dipolar potential only, calculated in the first Born approximation. Applying the Born approximation to the second term on the right hand side of Eq. (1), we find for $m=0$ and $l=l^{\prime}(l \geq 1)$

$$
a_{l l}=-\frac{2 D_{*}}{(2 l-1)(2 l+3)},
$$

and for $m=0$ and $l=l^{\prime}+2$

$$
a_{l, l-2}=-\frac{D_{*}}{(2 l-1) \sqrt{(2 l+1)(2 l-3)}} .
$$

For $l^{\prime}=2$ and $l=0$, e.g., Eq. (10) reduces to $a_{20}=$ $-D_{*} /(3 \sqrt{5})$, in agreement with Ref. [33]. The scattering lengths $a_{l-2, l}$ are equal to $a_{l, l-2}$, and all other generalized scattering lengths are zero. In Eqs. (9) and (10), $D_{*}$ denotes the dipole length, $D_{*}=\mu d^{2} / \hbar^{2}$. All non-zero scattering lengths $a_{l l^{\prime}}$ are negative, depend on $l$ and $l^{\prime}$, and are directly proportional to $d^{2}$. Furthermore, for fixed $D_{*}$, the absolute value of the non-zero $a_{l l^{\prime}}$ decreases with increasing angular momentum quantum number $l$, indicating that the coupling between different angular momentum channels decreases with increasing $l$. However, this decrease is quite slow and, in general, an accurate description of the two-dipole system requires that the convergence with increasing $l_{\max }$ be assessed carefully.

One can now show readily that the K-matrix elements $K_{l 0}^{l^{\prime} 0}$ of $V_{p p, r e g}$, calculated in the first Born approximation, with $a_{l l^{\prime}}$ given by Eqs. (9) and (10) coincide with the K-matrix elements $K_{l 0}^{l^{\prime} 0}$ of $V_{p p}$. This provides a simple check of the zerorange pseudo-potential construction and proofs that the prefactors of $V_{l l^{\prime}}$ are correct. In turn, this suggests that the applicability regimes of $V_{p p}$ and $V_{p p, r e g}$ are comparable, if the generalized scattering lengths $a_{l l^{\prime}}$ used to quantify the scattering strengths of $V_{l l^{\prime}}$ are approximated by Eqs. (9) and (10). The applicability regime of $V_{p p, r e g}$ may, however, be larger than that of $V_{p p}$ if the full energy-dependent K-matrix of a realistic potential is used instead.

To generalize the zero-range pseudo-potential defined in Eqs. (4) through (6) for projection quantum numbers $m=0$ to any $m$, only a few changes have to be made. In Eq. (5), the spherical harmonics $Y_{l 0}$ have to be replaced by $Y_{l m}$, and the generalized scattering lengths have to be defined through $\lim _{k \rightarrow 0}-K_{l m}^{l^{\prime} m^{\prime}} / k$. Correspondingly, Eqs. (9) and (10) become $m$-dependent.

\section{TWO DIPOLES UNDER EXTERNAL CONFINEMENT}

Section III A derives the implicit eigenequation for two dipoles interacting through the pseudo-potential under external harmonic confinement and Section $\amalg$ III analyzes the resulting eigen spectrum.

\section{A. Derivation of the eigenequation}

To determine the eigen energies of two aligned dipoles with $m=0$ under spherical harmonic confinement interacting through the zero-range potential $V_{p p, r e g}$, we expand the eigenfunctions $\Psi(\vec{r})$ in terms of the orthonormal harmonic oscillator eigen functions $R_{n_{i} l_{i}} Y_{l_{i} 0}$,

$$
\Psi(\vec{r})=\sum_{n_{i} l_{i}} c_{n_{i} l_{i}} R_{n_{i} l_{i}}(r) Y_{l_{i} 0}(\theta, \phi) .
$$

The pseudo-potential $V_{p p, r e g}$ enforces the proper boundary condition of $\Psi(\vec{r})$ at $r=0$, and thus determines the expansion coefficients $c_{n_{i} l_{i}}$. To introduce the key ideas we first consider $s$-wave interacting particles [28], for which the pseudopotential reduces to a single term, and then consider the general case, in which the pseudo-potential contains infinitely many terms.

Including only the term with $l$ and $l^{\prime}=0$ in Eq. (4), the Schrödinger equation becomes,

$$
\sum_{n_{i} l_{i}} c_{n_{i} l_{i}}\left(E_{n_{i} l_{i}}-E+V_{00}\right) R_{n_{i} l_{i}}(r) Y_{l_{i} 0}(\theta, \phi)=0
$$

where the $E_{n_{i} l_{i}}$ denote the eigenenergies of the non-interacting harmonic oscillator,

$$
E_{n_{i} l_{i}}=\left(2 n_{i}+l_{i}+\frac{3}{2}\right) \hbar \omega .
$$

In what follows, it is convenient to express the energy $E$ of the interacting system in terms of a non-integer quantum number v,

$$
E=\left(2 v+\frac{3}{2}\right) \hbar \omega .
$$

Multiplying Eq. (12) from the left with $R_{n_{o} l_{o}}^{*} Y_{l_{o} 0}^{*}$ with $l_{o}>$ 0 and integrating over all space, we find that the $c_{n_{i} l_{i}}$ with $l_{i}>0$ vanish. This can be understood readily by realizing that the $s$-wave pseudo-potential $V_{00}$, as discussed in detail in Sec. III only couples states with $l=l^{\prime}=0$. To determine the expansion coefficients $c_{n_{i}}$, we multiply Eq. (12) from the left with $R_{n_{0} 0}^{*} Y_{00}^{*}$ and integrate over all space. This results in

$$
c_{n_{o} 0}\left(2 n_{o}-2 v\right) \hbar \omega+R_{n_{o} 0}^{*}(0) g_{00} B_{0}=0,
$$


where $B_{0}$ denotes the result of the square bracket in Eq. (5),

$$
B_{0}=\left[\frac{\partial}{\partial r}\left(r \sum_{n_{i}=0}^{\infty} c_{n_{i} 0} R_{n_{i} 0}(r)\right)\right]_{r \rightarrow 0} .
$$

Note that $B_{0}$ is constant and independent of $n_{i}$. In Eq. 15, the $r$-independent term $R_{n_{o} 0}^{*}(0)$ arises from the radial integration over the $\delta$-function of the pseudo-potential. If we solve Eq. (15) for $c_{n_{o} 0}$ and plug the result into Eq. (16), the unknown constant $B_{0}$ cancels and we obtain an implicit eigenequation for $v$,

$$
1=g_{00}\left[\frac{\partial}{\partial r}\left(r \sum_{n_{i}=0}^{\infty} \frac{R_{n_{i} 0}^{*}(0) R_{n_{i} 0}(r)}{\left(2 v-2 n_{i}\right) \hbar \omega}\right)\right]_{r \rightarrow 0} .
$$

Using Eqs. (27) and (32) from the Appendix to simplify the term in square brackets, we obtain the well-known implicit eigenequation for two particles interacting through the $s$-wave pseudo-potential under spherical harmonic confinement [28],

$$
\frac{\Gamma\left(\frac{-E}{2 \hbar \omega}+\frac{1}{4}\right)}{2 \Gamma\left(\frac{-E}{2 \hbar \omega}+\frac{3}{4}\right)}-\frac{a_{00}}{a_{h o}}=0
$$

Here, $a_{h o}$ denotes the harmonic oscillator length, $a_{h o}=$ $\sqrt{\hbar /(\mu \omega)}$.

The derivation of the implicit eigenequation for two dipoles under external harmonic confinement interacting through the pseudo-potential with infinitely many terms proceeds analogously to that outlined above for the $s$-wave system. The key difference is that each $V_{l l^{\prime}}$ term in Eq. (5) with $l \neq l^{\prime}$ couples states with different angular momenta, resulting in a set of coupled equations for the expansion coefficients $c_{n_{i} l_{i}}$. However, since $V_{p p, r e g}$ for dipolar systems couples only angular momentum states with $\left|l-l^{\prime}\right| \leq 2$ [see, e.g., the discussion at the beginning of Sec. II and around Eqs. (9) and (10)], the coupled equations can, as we outline in the following, be solved analytically by including successively more terms in $V_{p p, r e g}$.

To start with, we plug the expansion given in Eq. (11) into Eq. (2), where the interaction potential $V_{\text {int }}$ is now taken to be the pseudo-potential $V_{p p, r e g}$ with infinitely many terms. To obtain the general equation for the expansion coefficients $c_{n_{i} l_{i}}$, we multiply as before from the left with $R_{n_{o} l_{o}}^{*} Y_{l_{o} 0}^{*}$ and integrate over all space,

$$
\begin{gathered}
c_{n_{o} l_{o}}\left(2 n_{o}+l_{o}-2 v\right) \hbar \omega+\left[\frac{R_{n_{o} l_{o}}^{*}(r)}{r^{l_{o}}}\right]_{r \rightarrow 0} \times \\
{\left[g_{l_{o}-2, l_{o}} B_{l_{o}-2}+g_{l_{o} l_{o}} B_{l_{o}}+g_{l_{o}+2, l_{o}} B_{l_{o}+2}\right]=0 .}
\end{gathered}
$$

Here, the $B_{l_{o}-2}, B_{l_{o}}$ and $B_{l_{o}+2}$ denote constants that are independent of $n_{i}$,

$$
B_{l_{o}}=\left[\frac{\partial^{2 l_{o}+1}}{\partial r^{2 l_{o}+1}}\left\{r^{l_{o}+1}\left(\sum_{n_{i}=0}^{\infty} c_{n_{i} l_{o}} R_{n_{i} l_{o}}(r)\right)\right\}\right]_{r \rightarrow 0}
$$

The three terms in the square bracket in the second line of Eq. (19) arise because the $V_{l^{\prime}-2, l^{\prime}}, V_{l^{\prime} l^{\prime}}$ and $V_{l^{\prime}+2, l^{\prime}}$ terms in the pseudo-potential $V_{p p \text {,reg }}$ couple the state $R_{n_{o} l_{o}}^{*} Y_{l_{o} 0}^{*}$, for $l^{\prime}=l_{o}$, with three components of the expansion for $\Psi$, Eq. (11). Importantly, the constants $B_{l_{o}-2}, B_{l_{o}}$ and $B_{l_{o}+2}$, defined in Eq. (20), depend on the quantum numbers $l_{o}-2, l_{o}$ and $l_{o}+2$, respectively, which implies that Eq. 19] defines a set of infinitely many coupled equations that determine, together with Eq. (20), the expansion coefficients $c_{n_{i}} l_{i}$. Notice that Eqs. (19) and (20) coincide with Eqs. (15) and (16) if we set $l_{o}=0$ and $g_{l l^{\prime}}=0$ if $l$ or $l^{\prime}>0$.

We now illustrate how Eqs. (19) and (20) can be solved for identical bosons, i.e., in the case where $l$ and $l^{\prime}$ are even (the derivation for identical fermions proceeds analogously). Our strategy is to solve these equations by including successively more terms in the coupled equations, or equivalently, in the pseudo-potential. As discussed above, if $a_{00}$ is the only nonzero scattering length, the eigenenergies are given by Eq. (18). Next, we also allow for non-zero $a_{20}, a_{02}$ and $a_{22}$, i.e., we consider $l$ and $l^{\prime} \leq 2$ in Eq. (4). In this case, the coefficients $c_{n_{i} 0}$ and $c_{n_{i} 2}$ are non-zero and coupled, but all $c_{n_{i} l_{i}}$ with $l_{i}>2$ are zero. Using the expressions for $B_{0}$ and $B_{2}$ given in Eq. (20), we decouple the equations. Finally, using Eqs. (27) and (32) from the Appendix, the eigenequation can be compactly written as

$$
t_{0}+\frac{q_{2}}{t_{2}}=0
$$

where

$$
t_{l}=\frac{\Gamma\left(\frac{-E}{2 \hbar \omega}+\frac{1}{4}-\frac{l}{2}\right)}{2^{2 l+1} \Gamma\left(\frac{-E}{2 \hbar \omega}+\frac{3}{4}+\frac{l}{2}\right)}-(-1)^{l} \frac{a_{l l}}{k^{2 l} a_{h o}^{2 l+1}},
$$

and

$$
q_{l}=-\frac{a_{l-2, l}^{2}}{k^{4 l-4} a_{h o}^{4 l-2}}
$$

Equation (21) can be understood as follows. If only $a_{00}$ is non-zero, it reduces to $t_{0}=0$, in agreement with Eq. (18). If only $a_{00}, a_{02}$ and $a_{20}$ are non-zero, Eq. (21) remains valid if $a_{22}$ in $t_{2}$ is set to zero. This shows that the term $q_{2}$ and the first term on the right hand side of $t_{2}$ arise due to the coupling between states with angular momenta 0 and 2 . The second term of $t_{2}$, in contrast, arises due to a non-zero $a_{22}$. Finally, for non-zero $a_{00}$ and $a_{22}$ but vanishing $a_{20}$ and $a_{02}$, Eq. (21) reduces to $t_{0} t_{2}=0$. In this case, we recover the eigenequations $t_{0}=0$ for $s$-wave interacting particles [28] and $t_{2}=0$ for $d$ wave interacting particles [32].

We now consider $l$ and $l^{\prime}$ values with up to $l_{\text {max }}=4$ in Eq. (4), i.e., we additionally allow for non-zero $a_{24}, a_{42}$ and $a_{44}$, and discuss how the solution changes compared to the $l_{\max }=2$ case. The equation for the expansion coefficients $c_{n_{i} 0}$ remains unchanged while that for $c_{n_{i} 2}$ is modified. Furthermore, the expansion coefficients $c_{n_{i} 4}$ are no longer zero. Consequently, we have three coupled equations, which can be decoupled, resulting in the following implicit eigenequation, $t_{0}+q_{2} /\left(t_{2}+q_{4} / t_{4}\right)=0$. In analogy to the $l_{\text {max }}=2$ case, the $q_{4}$ term and the first part on the right hand side of the $t_{4}$ term arise due to the "off-diagonal" scattering lengths $a_{24}$ and $a_{42}$, and the second term of $t_{4}$ arises due to the "diagonal" scattering length $a_{44}$. 
Next, let us assume that we have found the implicit eigenequation for the case where we include terms in Eq. (4) with $l$ and $l^{\prime}$ up to $l_{\max }-2$. If we now include terms with $l$ and $l^{\prime}$ up to $l_{\max }$, only the equations for the expansion coefficients $c_{n_{o} l_{o}}$ with $l_{o}=l_{\max }-2$ and $l_{\max }$ change; those for the expansion coefficients $c_{n_{o}} l_{o}$ with $l_{o} \leq l_{\max }-4$ remain unchanged. This allows the $l_{\max } / 2+1$ coupled equations for the expansion coefficients to be decoupled analytically using the results already determined for the case where $l$ and $l^{\prime}$ go up to $l_{\max }-2$. Following this procedure, we find the following implicit eigenequation

$$
T_{l_{\max }}=0
$$

where $T_{l_{\max }}$ itself can be written as a continued fraction. For identical bosons we find,

$$
T_{l_{\max }}=t_{0}+\frac{q_{2}}{t_{2}+\frac{q_{4}}{t_{4}+\cdots+\frac{q l_{\max }}{t_{\max }}}} .
$$

Taking $l_{\max } \rightarrow \infty$ gives the eigenequation for two identical bosons under spherical harmonic confinement interacting through $V_{p p, r e g}$ with infinitely many terms. For two identical fermions, Eqs. (22) through (25) remain valid if the subscripts $0,2, \cdots$ in Eq. (25) are replaced by $1,3, \cdots$.

The derived eigenequation reproduces the eigenenergies in the known limits. For the non-interacting case (all $a_{l l^{\prime}}=0$ ), the eigenenergies coincide with the eigenenergies of the harmonic oscillator, i.e., $E_{n l}=(2 n+l+3 / 2) \hbar \omega$, where $n=$ $0,1,2, \cdots$ and $l=0,2,4, \cdots$ (in the case of identical bosons) and $l=1,3, \cdots$ (in the case of identical fermions). The $k$ th levels, with energy $(2 k+3 / 2) \hbar \omega$ for bosons and $(2 k+5 / 2) \hbar \omega$ for fermions, has a degeneracy of $k+1, k=0,1, \cdots$. Nonvanishing $a_{l l^{\prime}}$ lead to a splitting of degenerate energy levels but leave the number of energy levels unchanged. If $a_{l l}$ is the only non-zero scattering length, the eigenequation reduces to that obtained for spherically symmetric pseudo-potentials with partial wave $l$ [32].

\section{B. Analysis of the energy spectrum}

This section analyses the implicit eigenequation, Eq. (24), derived in the previous section for the zero-range pseudopotential for $m=0$ and compares the resulting energy spectrum with that obtained for a shape-dependent model potential. The implicit eigenequation, Eq. (24), can be solved readily numerically by finding its roots in different energy regions. The solutions of the Schrödinger equation for the shape-dependent model potential are otained by expanding the eigenfunctions on a B-spline basis.

Lines in Figs. 1a) and (b) show the eigenenergies obtained by solving Eq. (24) for two identical bosons and two identical fermions, respectively, interacting through $V_{p s, r e g}(\vec{r})$ under external spherically symmetric harmonic confinement as a function of the dipole length $D_{*}$. In both panels, we assume that the interaction between the two dipoles is purely dipolar, i.e., in Fig. 1 a) we set $a_{00}=0$. The other scattering
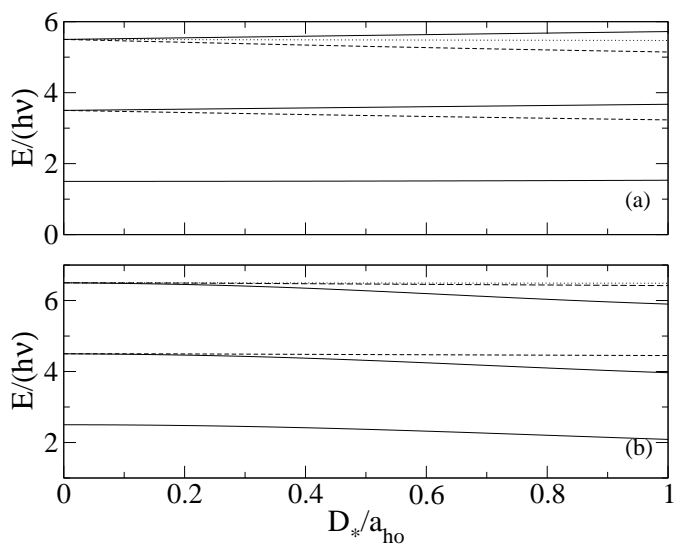

Figure 1: Relative eigenenergies $E$ for (a) two identical bosonic dipoles and (b) two identical fermionic dipoles interacting through $V_{p p \text {,reg }}$ [using $a_{00}=0$ in (a)] under spherical harmonic confinement as a function of $D_{*} / a_{h o}$. The line style indicates the predominant character of the corresponding eigenstates. In (a), a solid line refers to $l \approx 0$, a dashed line to $l \approx 2$, and a dotted line to $l \approx 4$; in $(\mathrm{b})$, a solid line refers to $l \approx 1$, a dashed line to $l \approx 3$, and a dotted line to $l \approx 5$.

lengths $a_{l l^{\prime}}$ are approximated by Eqs. (9) and (10). Interestingly, for identical bosons, the lowest gas-like level, which starts at $E=1.5 \hbar \omega$ for $D_{*}=0$, increases with increasing $D_{*}$. For identical fermions, in contrast, the lowest gas-like state decreases with increasing $D_{*}$.

In addition to obtaining the eigenenergies themselves, the pseudo-potential treatment allows the spectrum to be classified in terms of angular momentum quantum numbers. To this end, we solve the implicit eigenequation, Eq. (24), for increasing $l_{\text {max }}$, and monitor how the energy levels shift as additional angular momenta are included in $V_{p p, r e g}$. Since a level with approximate quantum number $l$ changes only little as larger angular momentum values are included in the pseudopotential, this analysis reveals the predominant character of each energy level. In Fig.1(a), the eigenfunctions of energies shown by solid, dashed and dotted lines have predominantly $l=0,2$ and 4 character, respectively. In Fig. 1 (b), the eigenfunctions of energies shown by solid, dashed and dotted lines have predominantly $l=1,3$ and 5 character, respectively. We find that the lowest excitation frequency between states with predominantly $l=0[l=1]$ character, increases [decreases] for identical bosons [fermions] with increasing $D_{*}$. These predictions can be verified directly experimentally.

To assess the accuracy of the developed zero-range pseudopotential treatment, we consider two interacting bosons with non-vanishing $s$-wave scattering length $a_{00}$. We imagine that the dipole moment of two identical polarized bosonic polar molecules is tuned by an external electric field. As the dipole moment $d$ is tuned, the $s$-wave scattering length $a_{00}$, which depends on the short-range and the long-range physics of the "true" interaction potential, changes. To model this situation, we solve the two-body Schrödinger equation, Eq. (2), numerically for a shape-dependent model potential with hardcore 



Figure 2: Panel (a) shows the relative energies $E$ for two aligned identical bosonic dipoles under external spherical harmonic confinement as a function of $D_{*} / a_{h o}$. Solid lines show the numerically determined energies obtained using $V_{\text {model }}$ with $b=0.0097 a_{h o}$. Crosses show the energies obtained using $V_{p p, r e g}$ with essentially infinitely many terms, and $a_{00}$ calculated for $V_{\text {model }}$. Panel (b) shows a blowup of the energy region around $E \approx 5.5 \hbar \omega$. Note that the horizontal axis in (a) and (b) are identical.

radius $b$ and long-range dipolar tail. In this case, $V_{i n t}$ is given by

$$
V_{\text {model }}(\vec{r})=\left\{\begin{array}{ll}
d^{2} \frac{1-3 \cos ^{2} \theta}{r^{3}} & \text { if } r \geq b \\
\infty & \text { if } r<b
\end{array} .\right.
$$

For $d=0$, the $s$-wave scattering length $a_{00}$ for $V_{\text {model }}$ is given by $b$. As the dipole length $D_{*}$ increases, $a_{00}$ goes through zero, and becomes negative. Just when the two-body potential supports a new bound state, $a_{00}$ goes through a resonance and becomes large and positive. As $D_{*}$ increases further, $a_{00}$ decreases. This resonance structure repeats itself with increasing $D_{*}$ (see Fig. 1 of Ref. [17]; note, however, that the lengths $a_{h o}$ and $D_{*}$ defined throughout the present work differ from those defined in Ref. [17]).

For the model potential $V_{\text {model }}, a_{00}$ depends on the ratio between the short-range and long-range length scales, i.e., on $b / D_{*}$. To compare the pseudo-potential energies and the energies for the model potential, we fix $b$ and calculate $a_{00}$ for each $D_{*}$ considered. The dipole-dependent $s$-wave scattering length is then used in the zero-range pseudo-potential $V_{p p, r e g}$. The other scattering lengths are, as before, approximated by the expressions given in Eqs. (9) and (10). Solid lines in Fig. 2(a) and (b) show the eigenenergies obtained for $V_{\text {model }}$ as a function of $D_{*}$. Crosses show the eigenenergies obtained for $V_{p p, r e g}$ using a value of $l_{\max }$ that results in converged eigenenergies. The overview spectrum shown in Fig. 2 (a) shows that one of the energy levels dives down to negative energies close to that $D_{*}$ value at which the two-body potential $V_{\text {model }}$ supports a new bound state. The blow-up, Fig.2(b), around $E \approx 5.5 \hbar \omega$ shows excellent agreement between the energies obtained using $V_{p p, r e g}$ (crosses) and those obtained using $V_{\text {model }}$ (solid lines); the maximum deviation for the energy range shown is $0.05 \%$.

As before, we can assign approximate quantum numbers to each energy level. At $D_{*} \ll a_{h o}$, the three energy levels around $E \approx 5.5 \hbar \omega$ have, from bottom to top, approximate quantum numbers $l=2,4$ and 0 . After two closely spaced avoided crossings around $D_{*} \approx 0.025 a_{h o}$, the assignment changes to $l=0,2$ and 4 (again, from bottom to top). If the maximum angular momentum $l_{\max }$ of the pseudo-potential is set to 2 , the energy level with approximate quantum number $l=4$ would be absent entirely. This illustrates that a complete and accurate description of the energy spectrum requires the use of a zero-range pseudo-potential with infinitely many terms. The energy of a state with approximate quantum number $l$ requires $l_{\max }$ to be at least $l$ for the correct degeneracy be obtained and at least $l+2$ for a quantitative description.

The sequence of avoided crossings at $D_{*} \approx 0.025 a_{h o}$ suggests an interesting experiment. Assume that the system is initially, at small electric field (i.e., small $D_{*} / a_{h o}$ ), prepared in the excited state with angular momentum $l \approx 0$ and $E \approx 5.52 \hbar \omega$. The electric field is then slowly swept across the first broad avoided crossing at $D_{*} \approx 0.019 a_{h o}$ to transfer the population from the state with $l \approx 0$ to the state with $l \approx 2$. We then suggest to sweep quickly across the second narrower avoided crossing at $D_{*} \approx 0.028 a_{h o}$ (the ramp speed must be chosen so minimize population transfer from the state with $l \approx 2$ to the state with $l \approx 4$ ). As in the case of $s$-wave scattering only [39], the time-dependent field sequence has to be optimized to obtain maximal population transfer. The proposed scheme promises to provide an efficient means for the transfer of population between states with different angular momenta and for quantum state engineering.

Figure 2 illustrates that the pseudo-potential treatment reproduces the eigenenergies of the shape-dependent model potential $V_{\text {model }}$. To further assess the validity of the pseudopotential treatment, we now consider two interacting bosonic dipoles for which the dipolar interaction is dominant, i.e., we consider $a_{00}=0$. For $V_{\text {model }}$ with $b=0.0031 a_{h o}$, we determine a set of $D_{*}$ values at which $a_{00}=0$. Note that the number of bound states with predominantly s-wave character increases by one for each successively larger $D_{*}$. Crosses in Figs. 3(a)-(c) show the eigenenergies for $V_{\text {model }}$ with $a_{00}=0$ as a function of $D_{*}$ in the energy ranges around 1.5, 3.5 and $5.5 \hbar \omega$. For comparison, lines show the eigenenergies obtained for the regularized pseudo-potential with $a_{00}=0$. As in Fig. 1, the linestyle indicates the predominant character of the energy levels (solid line: $l \approx 0$; dashed line: $l \approx 2$; and dotted line: $l \approx 4)$. The agreement between the energies obtained for the pseudo-potential with $a_{l l^{\prime}}$ given by Eqs. (9) and (10) and for the model potential for small $D_{*}$ is very good, thus validating the applicability of the pseudo-potential treatment. The agreement becomes less good, however, as $D_{*}$ increases. This can be explained readily by realizing that the dipole length $D_{*}$ approaches the harmonic oscillator length $a_{h o}$.

In general, the description of confined particles interacting through zero-range pseudo-potentials is justified if the characteristic lengths of the two-body potential are smaller than the characteristic length of the confining potential. For example, in the case of $s$-wave interactions only, the van der Waals 


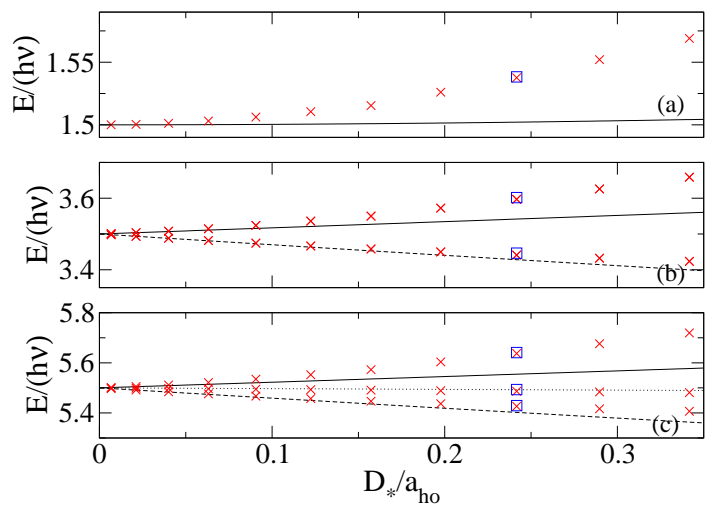

Figure 3: Crosses show the relative eigenenergies $E$ as a function of $D_{*} / a_{h o}$ for two identical bosons with $a_{00}=0$ interacting through $V_{\text {model }}$ with $b=0.0031 a_{\text {ho }}$ in three different energy regions. Lines show $E$ for two identical bosons with $a_{00}=0$ interacting through $V_{p s, \text { reg }}$ with $a_{l l^{\prime}}$ given by Eq. (9) and (10). As in Fig. 11a) solid, dashed and dotted lines show the energies of levels characterized by approximate quantum numbers $l \approx 0,2$ and 4 . The agreement between the crosses and the lines is good at small $D_{*} / a_{h o}$ but less good at larger $D_{*} / a_{h o}$. Squares show the eigenenergies obtained for the energy-dependent pseudo-potential at $D_{*}=0.242 a_{h o}$; the agreement between the squares and the crosses is excellent, illustrating that usage of the energy-dependent K-matrix greatly enhances the applicability regime of $V_{p p, r e g}$.

length has to be smaller than the oscillator length [29, 30]. The model potential $V_{\text {model }}$ is characterized by a short-range length scale, the hardcore radius $b$, and the dipole length $D_{*}$; in Fig. 3, it is the relatively large value of $D_{*} / a_{h o}$ that leads, eventually, to a break-down of the pseudo-potential treatment. As in the case of spherical interactions, the breakdown can be pushed to larger $D_{*}$ values by introducing energy-dependent generalized scattering lengths $a_{l l^{\prime}}(k)$, defined through $-K_{l 0}^{l^{\prime} 0}(k) / k$ for $m=0$, and by then solving the eigenequation, Eq. (24), self-consistently [29, 30].

Figure 4 shows three selected scattering lengths $a_{l l^{\prime}}(k)$ for the model potential $V_{\text {model }}$ with $D_{*}=78.9 \mathrm{~b}$ as a function of energy. This two-body potential supports eight bound states with projection quantum number $m=0$, which have predominantly $s$-wave character. Both energy and length in Fig. 4 are expressed in oscillator units to allow for direct comparison with the data shown in Fig. 3. The scattering length $a_{00}(k)$, shown by a solid line in Fig. 4, is zero at zero energy and increases with increasing energy. Both $a_{20}(k)$ (dashed line) and $a_{22}(k)$ (dash-dotted line) are negative. Their zero-energy values coincide with those calculated in the Born approximation (horizontal dotted lines).

Using these energy-dependent $a_{l l^{\prime}}(k)$ to parametrize the strengths of the pseudo-potential and solving the eigenequation, Eq. (24), self-consistently, we obtain the squares in Fig. 3 The energies for $V_{p p, r e g}$ with energy-dependent $a_{l l^{\prime}}$ (squares) are in much better agreement with the energies obtained for the model potential (crosses) than the energies ob-

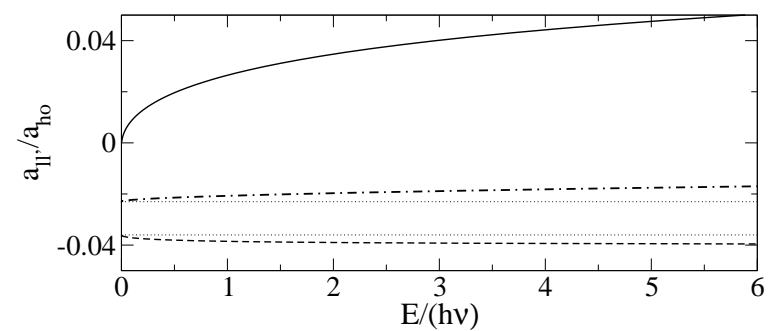

Figure 4: Energy-dependent scattering lengths $a_{00}(k)$ (solid line), $a_{20}(k)$ (dashed line) and $a_{22}(k)$ (dash-dotted line) for the model potential $V_{\text {model }}$ with $D_{*}=78.9 \mathrm{~b}$ as a function of the relative energy $E$. In oscillator units, $V_{\text {model }}$ is characterized by $b=0.0031 a_{h o}$ and $D_{*}=0.242 a_{h o}$. For comparison, horizontal dotted lines show the energy-independent scattering lengths $a_{22}$, Eq. (9), and $a_{20}$, Eq. (10), calculated in the first Born approximation.

tained using the energy-independent $a_{l l^{\prime}}$ to parametrize the pseudo-potential (lines). This suggests that the applicability regime of the regularized zero-range pseudo-potential can be extended significantly by introducing energy-dependent scattering lengths. Since the proper treatment of resonant interactions within the regularized zero-range pseudo-potential requires that the energy-dependence of the generalized scattering lengths be included, future work will address this issue in more depth.

\section{SUMMARY}

This paper applies a zero-range pseudo-potential treatment to describe two interacting dipoles under external spherically harmonic confinement. Section $\amalg$ introduces the regularized zero-range pseudo-potential $V_{p p, r e g}$ used in this work, which was first proposed by Derevianko [33, 34]. Particular emphasis is put on developing a simple interpretation of the individual pieces of the pseudo-potential. Furthermore, we clearly establish the connection between $V_{p p, r e g}$ and the pseudo-potential $V_{p p}$, which is typically employed within a mean-field framework. We argue that the applicability regime of these two pseudo-potentials is comparable if the scattering strengths of $V_{p p, r e g}$, calculated in the first Born approximation, are chosen so as to reproduce those of $V_{p p}$.

We then use the regularized zero-range pseudo-potential to derive an implicit eigen equation for two dipoles under external confinement, a system which can be realized experimentally with the aid of optical lattices. In deriving the implicit eigenequation, we again put emphasis on a detailed understanding of how the solution arises, thus developing a greater understanding of the underlying physics. The implicit eigenequation can be solved straightforwardly, and allows for a direct classification scheme of the resulting eigenspectrum. By additionally calculating the eigen energies for two dipoles interacting through a finite range model poten- 
tial numerically, we assess the applicability of the developed zero-range pseudo-potential treatment. We find good agreement between the two sets of eigenenergies for small $D_{*}$ and quantify the deviations as $D_{*}$ increases. Finally, we show that the validity regime of $V_{p p, r e g}$ can be extended by parametrizing the scattering strengths of $V_{p p, r e g}$ in terms of the energydependent K-matrix calculated for a realistic model potential. This may prove useful also when describing resonantly interacting dipoles.

At first sight it may seem counterintuitive to replace the long-range dipolar interaction by a zero-range pseudopotential. However, if the length scales of the interaction potential, i.e., the van der Waals length scale characterizing the short-range part and the dipole length characterizing the longrange part of the potential, are smaller than the characteristic length of the trap $a_{h o}$, this approach is justified since the zerorange pseudo-potential is designed to reproduce the K-matrix elements of the "true" interaction potential. This is particularly true if the pseudo-potential is taken to contain infinitely many terms, as done in this work.

In summary, this paper determines the eigenenergies of two interacting dipoles with projection quantum number $m=0$. The applied zero-range pseudo-potential treatment is validated by comparing the resulting eigenenergies with those obtained numerically for a shape-dependent model potential. The analysis presented sheds further light on the intricate properties of angle-dependent scattering processes and their description through a regularized zero-range pseudo-potential with infinitely many terms. The calculated energy spectrum may aid on-going experiments on dipolar Bose and Fermi gases.

Acknowledgements: KK and DB acknowledge support by the NSF through grant PHY-0555316 and JLB by the DOE.

\section{APPENDIX}

In this Appendix, we evaluate the following infinite sum,

$$
C_{l}=\left[\frac{\partial^{2 l+1}}{\partial r^{2 l+1}}\left\{r^{l+1} \sum_{n=0}^{\infty} \frac{\left[\frac{R_{n l}^{*}(r)}{r^{l}}\right]_{r \rightarrow 0} R_{n l}(r)}{2\left(\mathrm{v}-n-\frac{l}{2}\right) \hbar \omega}\right\}\right]_{.}
$$

Writing the radial harmonic oscillator functions $R_{n l}(r)$ in terms of the Laguerre polynomials $L_{n}^{(l+1 / 2)}$,

$$
\begin{gathered}
R_{n l}(r)=\sqrt{\frac{2^{l+2}}{(2 l+1) ! ! \pi^{1 / 2} L_{n}^{(l+1 / 2)}(0) a_{h o}^{3}}} \times \\
\quad \exp \left(-\frac{r^{2}}{2 a_{h o}^{2}}\right)\left(\frac{r}{a_{h o}}\right)^{l} L_{n}^{(l+1 / 2)}\left(r^{2} / a_{h o}^{2}\right),
\end{gathered}
$$

we find

$$
\left[\frac{R_{n l}(r)}{r^{l}}\right]_{r \rightarrow 0}=\sqrt{\frac{2^{l+2} L_{n}^{(l+1 / 2)}(0)}{(2 l+1) ! ! \pi^{1 / 2} a_{h o}^{2 l+3}}} .
$$

Using Eqs. (28) and 29), the $C_{l}$ can be rewritten as

$$
\begin{array}{r}
C_{l}=\frac{2^{l+1}}{(2 l+1) ! ! \pi^{1 / 2} a_{h o}^{2 l+3}} \times \\
{\left[\frac{\partial^{2 l+1}}{\partial r^{2 l+1}}\left(\exp \left(\frac{-r^{2}}{2 a_{h o}^{2}}\right) r^{2 l+1} \sum_{n=0}^{\infty} \frac{L_{n}^{(l+1 / 2)}\left(\left(\frac{r}{a_{h o}}\right)^{2}\right)}{\left(v-n-\frac{l}{2}\right) \hbar \omega}\right)\right]_{r \rightarrow 0}}
\end{array}
$$

We evaluate the infinite sum in Eq. 30 using the properties of the generating function [40],

$$
\begin{gathered}
\sum_{n=0}^{\infty} \frac{L_{n}^{(l+1 / 2)}\left(\left(r / a_{h o}\right)^{2}\right)}{v-n-\frac{l}{2}}= \\
-\Gamma(-v+l / 2) U\left(-v+l / 2, l+3 / 2,\left(r / a_{h o}\right)^{2}\right) .
\end{gathered}
$$

Using Eq. (31) together with the small $r$ behavior of the hypergeometric function $U$ [41], the expression for the $C_{l}$ reduces to

$$
C_{l}=\frac{(-1)^{l} 2^{2 l+2}(2 l) ! !}{(2 l+1) ! !} \frac{\Gamma\left(-v+\frac{l}{2}\right)}{\Gamma\left(-v-\frac{l+1}{2}\right)} \frac{1}{\hbar \omega a_{h o}^{2 l+3}}
$$

[1] A. Griesmaier et al., Phys. Rev. Lett. 94, 160401 (2005).

[2] J. Stuhler et al., Phys. Rev. Lett. 95, 150406 (2005).

[3] S. Y. T. van der Meerakker et al., Phys. Rev. Lett. 94, 023004 (2005).

[4] J. R. Bochinski, E. R. Hudson, H. J. Lewandowski, and J. Ye, Phys. Rev. A 94, 043410 (2004).

[5] D. Wang et al., Phys. Rev. Lett. 93, 243005 (2004).

[6] A. J. Kerman, J. M. Sage, S. Sainis, T. Bergeman, and D. DeMille, Phys. Rev. Lett. 92, 153001 (2004).

[7] D. H. J. O’Dell, S. Giovanazzi, and G. Kurizki, Phys. Rev. Lett. 90, 110402 (2003).

[8] L. Santos, G. V. Shlyapnikov, and M. Lewenstein, Phys. Rev. Lett. 90, 250403 (2003).

[9] S. Ronen, D. C. E. Bortolotti, and J. L. Bohn, cond-mat/0607704
[10] L. Santos, G. V. Shlyapnikov, P. Zoller, and M. Lewenstein, Phys. Rev. Lett. 85, 1791 (2000).

[11] S. Yi and L. You, Phys. Rev. A 61, 041604(R) (2000).

[12] K. Goral, K. Rzazewski, and T. Pfau, Phys. Rev. A 61, 051601(R) (2000).

[13] J.-P. Martikainen, M. Mackie, and K.-A. Suominen, Phys. Rev. A 64, 037601 (2001).

[14] S. Yi and L. You, Phys. Rev. A 63, 053607 (2001).

[15] K. Góral and L. Santos, Phys. Rev. A 66, 023613 (2002).

[16] S. Ronen, D. C. E. Bortolotti, D. Blume, and J. L. Bohn, Phys. Rev. A 74, 033611 (2006).

[17] D. C. E. Bortolotti, S. Ronen, J. L. Bohn, and D. Blume, Phys. Rev. Lett. 97, 160402 (2006).

[18] G. K. Brennen, C. M. Caves, P. S. Jessen, and I. H. Deutsch, Phys. Rev. Lett. 82, 1060 (1999). 
[19] D. Jaksch et al., Phys. Rev. Lett. 85, 2208 (2000).

[20] D. DeMille, Phys. Rev. Lett. 88, 067901 (2002).

[21] G. K. Brennen, I. H. Deutsch, and C. J. Williams, Phys. Rev. A 65, 022313 (2002).

[22] K. Góral, L. Santos, and M. Lewenstein, Phys. Rev. Lett. 88, 170406 (2002).

[23] B. Damski et al., Phys. Rev. Lett. 90, 110401 (2003).

[24] R. Barnett, D. Petrov, M. Lukin, and E. Demler, Phys. Rev. Lett. 96, 190401 (2006).

[25] A. Micheli, G. K. Brennen, and P. Zoller, Nature Physics 2, 341 (2006).

[26] E. Fermi, Nuovo Cimento 11, 157 (1934).

[27] K. Huang and C. N. Yang, Phys. Rev. 105, 767 (1957).

[28] T. Busch, B.-G. Englert, K. Rzążewski, and M. Wilkens, Foundations of Phys. 28, 549 (1998).

[29] D. Blume and C. H. Greene, Phys. Rev. A 65, 043613 (2002).

[30] E. L. Bolda, E. Tiesinga, and P. S. Julienne, Phys. Rev. A 66, 013403 (2002).

[31] K. Kanjilal and D. Blume, Phys. Rev. A 70, 042709 (2004).
[32] R. Stock, A. Silberfarb, E. L. Bolda, and I. H. Deutsch, Phys. Rev. Lett. 94, 023202 (2005).

[33] A. Derevianko, Phys. Rev. A 67, 033607 (2003).

[34] A. Derevianko, Phys. Rev. A 72, 039901(E) (2005).

[35] S. Yi and L. You, Phys. Rev. Lett. 92, 193201 (2004).

[36] I. Reichenbach, A. Silberfarb, R. Stock, and I. H. Deutsch, Phys. Rev. A 74, 042724 (2006).

[37] Z. Idziaszek and T. Calarco, Phys. Rev. Lett. 96, 013201 (2006).

[38] Scattering theory of waves and particles, 2nd edition, by R. G. Newton, Dover Publications Inc., New York (1982).

[39] J. Dunn et al., Phys. Rev. A 71, 033402 (2005).

[40] Handbook of Mathematical Functions, edited by M. Abramowitz and I. E. Stegun, Department of Commerce, Washington, DC, 1972, entry (22.9.15) in Table 22.9 and and entry (13.2.5).

[41] Handbook of Mathematical Functions, edited by M. Abramowitz and I. E. Stegun, Department of Commerce, Washington, DC, 1972, entries (13.1.2) and (13.1.3). 